\title{
Experimental Validation and Comparisons of Active Filtering Strategies
}

\author{
Fernando Pinhabel Marafão
}

Sigmar Maurer Deckmann

\author{
School of Electrical and Computer Engineering \\ University of Campinas - UNICAMP \\ Campinas - SP, 13083-852 - Brazil \\ phone: +5519 37883710, fax:+55 1932891395 , \\ e-mail: fmarafao@ieee.org, sigmar@dsce.fee.unicamp.br
}

\begin{abstract}
This paper deals with different active filtering strategies and digital techniques that can be applied to their implementation. The central idea is based on an alternative method to evaluate the disturbing voltage and current components through selective decompositions. Those decompositions, applied directly on voltage and current signals, are used to identify the distorting, unbalancing and non-active components and can be used to compensate either non-active power, power oscillations, harmonics and asymmetries in a different way as provided by the well known $p q$ Theory. Four compensation strategies are discussed and compared for solving typical power quality problems. Simulation and experimental results illustrate the main differences among them, when facing non-linear, unsymmetrical and variable loads under generic voltage conditions. According to these results, a shunt active filtering is even capable to improve the PCC voltages under critical conditions or can allow full compensation of a set of chosen harmonics, even if the active filter has limited bandwidth
\end{abstract}

Key words: Active filters, compensation strategies, current compensation, instantaneous power theory, power quality, selective compensation.

\section{Introduction}

Several researches concerning active filtering have been carried out in the last twenty years. Most of them directed to the active filters topologies [1], their compensation strategies [2-9] and the corresponding voltage and current controllers [10].

Regarding to the topologies, among several active structures, the shunt active power filter has been the most useful power conditioner. This can be easily explained based on its simple scheme and the vast sort of compensation strategies that can be implemented using the same equipment. Such filter is able to compensate most of power quality problems related to current deterioration, such as waveform distortion, unbalances, non-active or reactive currents, power factor, etc.

Concerning with the compensation strategies, many authors have been working on the issue of proposing new control methods in order to improve the active filter compensation results or even to simplify its implementation. The most focused methods are based on the $p q$ Theory or any of its adaptations [2], but it is very important to point out that there are many other alternatives, which can be, theoretically, even more flexible than the previous one and can make easier the understanding of instantaneous power components and the potential of power quality conditioning [11-17].

So, the first goal of this paper is to present a sequential decomposition procedure to be applied on measured voltages and currents, in order to identify, selectively, the ideal balanced and sinusoidal voltages and the in-phase sinusoidal currents of the power system (and consequently, different disturbance signals). These decompositions are symbolically shown in Fig.1. Accordingly, several alternative control methods for active filtering or power conditioning can also be drawn from this methodology.

Moreover, the compensation based on such methodology is quite flexible, since the decompositions enable the selective identification and elimination of the disturbing effects. As presented in [13], this methodology can be considered a combination of Fortescue's and Fryze's classic theories, along with the use of instantaneous vector algebra concepts. It can be used to formulate an instantaneous power theory under generic conditions and it can also be applied to power quality monitoring. In this work, the decompositions will be used to calculate the active filter control variables, independent of periodicity, distortions, unbalances, number of phases and the presence of neutral wire.

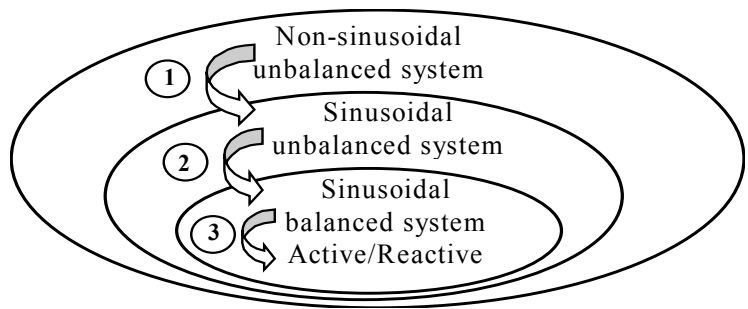

Fig.1 - Sequential decompositions principle. 
Since the correct choice of the compensation strategy is one of the most important requirements for power quality improvement [3,7], section 4 presents various considerations about positive and negative aspects of some techniques to control active filters, particularly shunt filters, in order to compensate non-active power components, harmonics, unbalances, active power oscillations, power factor, etc.

The strategies have been explored are the Sinusoidal Source Current Method, which ensures that the loads drain only sinusoidal and balanced currents from the power grid; the Resistive Load Synthesis Method, which makes the power system to "sense" the non-linear loads as a pure resistive balanced load, independently of the utility voltages; and the Selective Harmonic Compensation Method, which can be designed to compensate just the more significant load's harmonic frequencies, ensuring reduced power converters and better control and dynamic behavior to the active filters.

Simulation and experimental results with a $5,5 \mathrm{kVA}$ prototype validate the theoretical discussions and demonstrate the relevance of the proposed selective identification method and the correct choice of the compensation strategy for each specific power quality problem.

\section{Sequential Decompositions and Selective Disturbance Identification Method}

As suggested in Fig. 1, three main signal decompositions can be used in order to obtain the ideal voltage and current components in the sense of pure and clean power delivering and consumption.

The first decomposition is necessary to separate the fundamental frequency $(50 / 60 \mathrm{~Hz})$ from all other distorting components, which are imposed by non-linear loads and switching devices. The second decomposition is necessary to identify the positive sequence comprising the fundamental voltages and currents, in order to quantify the unbalancing components, responsible for power oscillations at the fundamental frequency. The third decomposition is necessary to separate the active and reactive currents of the fundamental, balanced components, in order to evaluate the demand for active or non-active power. A simplified diagram of such procedure is depicted in Fig. 2.

From the power quality point of view, if the proposed decompositions are able to identify the ideal voltage and current components, they are even able (by simple arithmetical expressions) to determine the voltage and current parcels responsible for power disturbing effects. Consequently, it is possible to identify the main

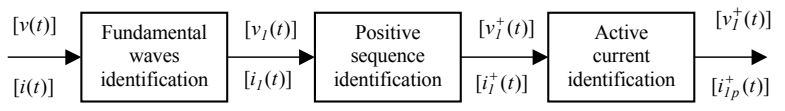

Fig. 2. Sequential decomposition procedure. power quality deterioration causes and to develop methods and techniques to improve the power quality requirements demanded by sensitive costumers or to provide minimum losses.

Next sections describe each decomposition procedure for a generic system with non-sinusoidal and unbalanced voltages feeding a generic non-resistive load. For simplicity, the voltage and current variables are assigned as instantaneous multidimensional vectors ( $\boldsymbol{v}$ e $\boldsymbol{i}$ ), and represented in this text by bold variables as follows:

$$
\boldsymbol{v}=\left[\begin{array}{lll}
v_{a} & v_{b} & v_{c}
\end{array}\right] \quad \boldsymbol{i}=\left[\begin{array}{lll}
i_{a} & i_{b} & i_{c}
\end{array}\right]
$$

\section{A. Fundamental Wave Identification $-1^{\text {st }}$ Decomposition}

The first decomposition breaks the measured signals ( $\boldsymbol{v}$ and $\boldsymbol{i}$ ), into their fundamental components $\left(\boldsymbol{v}_{l}\right.$ and $\left.\boldsymbol{i}_{l}\right)$ and residual signals $\left(\boldsymbol{v}_{\text {res }}\right.$ and $\left.\boldsymbol{i}_{\text {res }}\right)$, which correspond to the voltages and currents waveform distortions (harmonics and inter-harmonics).

$$
v=v_{1}+v_{r e s} \quad i=i_{1}+i_{r e s}
$$

The resulting residual components can be used in different ways. For example, they could be used to monitor the Total Harmonic Distortion (THD) as proposed in [18], or as the reference signal of an active filter in order to compensate just the voltage or current waveform distortion. Regarding the harmonic elimination, an interesting solution for selective harmonic compensation is also discussed further in this paper.

\section{B. Positive Sequence Identification $-2^{\text {nd }}$ Decomposition}

The second decomposition provides the identification of the fundamental voltages and currents unbalances. Starting from the identified instantaneous fundamental vectors $\left(\boldsymbol{v}_{l}\right.$ and $\left.\boldsymbol{i}_{l}\right)$, which may be unbalanced, the second decomposition realizes the calculation of the instantaneous positive sequence components:

$$
\boldsymbol{v}_{\boldsymbol{1}}^{+}=\left[\begin{array}{llll}
v_{1 a}^{+} & v_{1 b}^{+} & v_{1 c}^{+}
\end{array}\right] \quad \boldsymbol{i}_{\boldsymbol{1}}^{+}=\left[\begin{array}{lll}
i_{1 a}^{+} & i_{1 b}^{+} & i_{1 c}^{+}
\end{array}\right]
$$

The difference between the voltages and currents from (1) and (3) correspond to the distortion and unbalance parcels of $\boldsymbol{v}$ and $\boldsymbol{i}$, and hence, this difference could be considered as a correction signal for active filters with the purpose of eliminating both harmonics and unbalancing effects of the system. This should reduce losses and oscillating effects, besides increasing the installation power factor [19].

Accordingly, the ideal compensation of voltages at the Point of Common Coupling (PCC) could utilize the instantaneous disturbing voltages represented by:

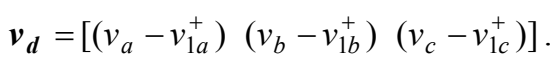

On the other hand, if one needs to compensate only the fundamental unbalance, the difference between (3) 
and the fundamental waves (2) after the first decomposition ( $v_{l}$ and $i_{1}$ ) could be used as the error signals to be eliminated.

\section{Lagrange Multiplier $-3^{\text {rd }}$ Decomposition}

A third decomposition is necessary to identify the parcel of the instantaneous fundamental current, which is proportional to the balanced sinusoidal voltages, thus representing the current fed to a pure resistive load, supplied by the sinusoidal and balanced voltages $v_{l}^{+}$. The method used in this decomposition is based on the Lagrange Multiplier or Orthogonal Decomposition [11$13,23]$. The ideal current parcel is defined as the positive sequence fundamental active current and it is expressed by means of the instantaneous vector:

$$
\boldsymbol{i}_{1 p}^{+}=\left[i_{1 a p}^{+} i_{1 b p}^{+} i_{1 c p}^{+}\right]=\frac{p_{1}^{+}}{v_{1 a}^{+}+v_{1 b}^{+}+v_{1 c}^{+}} \cdot \boldsymbol{v}_{1}^{+},
$$

where $p_{1}^{+}=\boldsymbol{v}_{1}^{+} \cdot \boldsymbol{i}_{1}^{+}=v_{1 a}^{+} \cdot i_{1 a}^{+}+v_{1 b}^{+} \cdot i_{1 b}^{+}+v_{1 c}^{+} . i_{1 c}^{+}$.

In the same way of definition (4), the difference between the instantaneous measured currents (1) and the evaluated ideal currents (5) defines the disturbing currents $i_{d}$, which are responsible for waveform distortions, phase current unbalances and also for the non-active power, which does not contribute to the unidirectional power exchange between power supplies and loads, which means that it does not realize useful work $[11,13,15]$. The disturbing current vector $i_{d}$ can be represented by:

$$
\boldsymbol{i}_{\boldsymbol{d}}=\left[\left(i_{a}-i_{1 a p}^{+}\right)\left(i_{b}-i_{1 b p}^{+}\right)\left(i_{c}-i_{1 c p}^{+}\right)\right]
$$

\section{Selective Identification Principle}

The proposed selective identification methodology is based on using the discussed decompositions either sequentially, as described above, or combining the Lagrange Multiplier in different positions, as depicted in Fig. 3. Thus, it is possible to evaluate ideal or disturbing voltage and current components and subsequently, to define the desired compensation or monitoring reference signals.

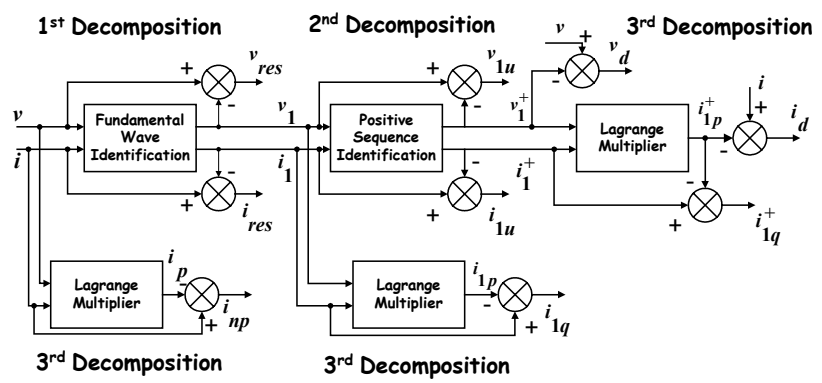

Fig. 3 - Selective Disturbing Identification Methodology.

The following index notation should be applied:
1 - fundamental frequency components;

res - residual or distorting components;

$+\quad$ - positive sequence components;

$\boldsymbol{u} \quad$ - unbalanced components;

p - active current component;

np - non-active current component;

$\boldsymbol{q} \quad$ - reactive current component;

d - disturbing components.

It is also important to stress that each of the mentioned decompositions, particularly the first two, can be implemented in different ways when using digital processing techniques. This will be detailed in the next section.

\section{Digital Implementation Techniques}

In the last few years, the authors have been investigating different digital implementations for each of the idealized decompositions and depending on the chosen digital technique, the results can be quite different. This is especially important to dynamical analysis and steady state errors evaluation. Moreover, depending on the digital system (DSP, FPGA, fixedpoint, floating-point, etc), one or another method may not ensure the decomposition's desired performance.

\section{A. $1^{\text {st }}$ Decomposition}

The first decomposition could be implemented for example in two different ways:

\section{1) Using an IIR notch filter - band-pass algorithm:}

As proposed in [18], an Infinite Impulse Response (IIR) notch filter can be used as in diagram of Fig. 4, in order to evaluate the fundamental and residual components of a generic waveform. The very narrowband filter response may ensure a good filtering performance, however, some attention must be directed to the cases of fundamental frequency variations, as well as when using fixed-point digital implementations.

The former point should be solved by means of some sort of adaptative IIR implementation, while the latter case requires, e.g., unusual solutions like Delta Operator's discretization procedure [20].

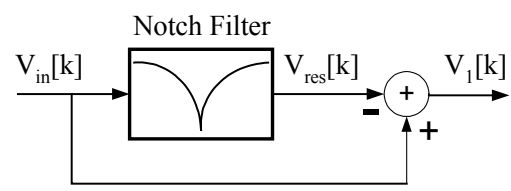

Fig. 4 - Band-pass algorithm based on notch filter.

\section{2) Using a FIR band-pass filter-DCT filter:}

An alternative to implement the first decomposition is to use a Finite Impulse Response (FIR) filter by means of a Discrete Cosine Transform (DCT) algorithm [24]. The basic equation to a DCT filter is shown in (7) and it could be implemented using circular buffers: 


$$
v_{1}=\frac{2}{N} \sum_{i=0}^{N-1} v(i) \cdot \cos \left[\frac{2 \pi}{N} \cdot i\right],
$$

where $i$ is the sampling counter and $N$ the number of samples per fundamental cycle.

As the previous IIR filter, the DCT is also sensitive to frequency deviations and one solution in this case, could be use a Phase Locked Loop (PLL) system to adjust the sampling frequency in order to ensure that $N$ is always the same. Other solutions are based on adaptative FIR filters [20]. Anyway, it is important to notice that this solution is quite better concerning to fixed-point implementations than those using IIR filters, at the cost of a relatively more complex computational implementation.

\section{B. $2^{\text {nd }}$ Decomposition}

The positive sequence identification can also be done in different ways, but basically, the first decomposition is also required.

\section{1) Instantaneous Fortescue application:}

As proposed in [13], the Fortescue's transformation can be implemented in time domain. Assuming 3-phase voltages of sequence $a b c$, then the angular shifting operator $a=e^{j 120^{\circ}}=1 \angle 120^{\circ}$ may be substituted by a time shift ahead, equivalent to one third of the fundamental period $(T / 3)$. Thus the fundamental positive component, referenced to phase $a$, can be expressed as:

$$
\begin{aligned}
& v_{1}^{+}=\frac{1}{3}\left[v_{1 a}+v_{1 b} \angle 120^{\circ}+v_{1 c} \angle-120^{\circ}\right], \\
& v_{1}^{+}=\frac{1}{3}\left[v_{1 a}(t)+v_{1 b}\left(t+\frac{T}{3}\right)+v_{1 c}\left(t-\frac{T}{3}\right)\right] .
\end{aligned}
$$

Note that the voltages sum up at different time instants. To be strictly valid, this requires steady state conditions. But due to the use of the notch filter, the fundamental voltage resulting from the first decomposition, presents slow transient response, thus permitting to realize the sum (8.b) by making the following substitution:

$$
v_{1 b}\left(t+\frac{T}{3}\right) \cong v_{1 b}\left(t-\frac{2 T}{3}\right),
$$

so, only past values may be used to obtain the positive sequence of the slowly changing fundamental voltages. The dynamic response in this case is certainly defined by the fundamental component evaluation.

\section{2) Modified DCT filter:}

It is also possible to use a modified DCT, in order to get the positive sequence components [20]. In this case the transient response of the positive sequence detector is exactly one fundamental cycle.

\section{3) Positive sequence detector using PLL:}

Using a digital PLL algorithm to ensure fundamental frequency tracking and a moving average filter to calculate the correct amplitude of the instantaneous positive sequence, the authors have proposed a novel positive sequence detector [22]. It is not based on the Fortescue's definitions, but exactly matches its stead state results. Diagram of Fig. 5 illustrate the proposed algorithm. The dynamic response in this case is also limited in one fundamental cycle.

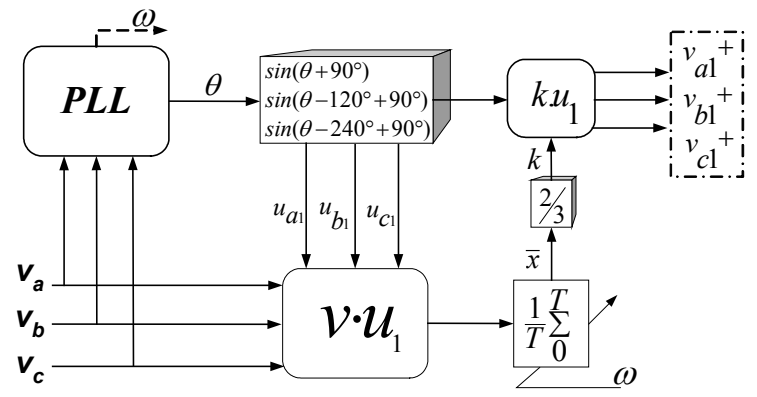

Fig. 5 - Novel algorithm for Positive Sequence Detection.

\section{Selective Harmonic Identification}

As previously mentioned, the first decomposition is responsible to identify the residual or distorting components of voltage or current signals, which could be used to harmonic compensation by means of an active filter. Indeed, recent works $[5,8,9]$ have pointed out the advantages of selective harmonic compensation, which does not force the active filter to eliminate all the distorting components $\left(v_{\text {res }}\right.$ and $\left.i_{\text {res }}\right)$, but only the more significant ones. This ensures reduced power converter requirements, lower switching frequencies, without harming the harmonic compensation.

This strategy is implemented by means of repetitive controller (Fig. 6) and the desired harmonics to be eliminated are selected using the DCT discrete filter [9]:

$$
F_{D C T}(z)=\frac{2}{N} \sum_{i=0}^{N-1}\left(\sum_{h \in N_{h}} \cos \left[\frac{2 \pi}{N} h(i)\right]\right) z^{-i},
$$

where $N_{h}$ is the set of selected harmonic frequencies $(h)$.

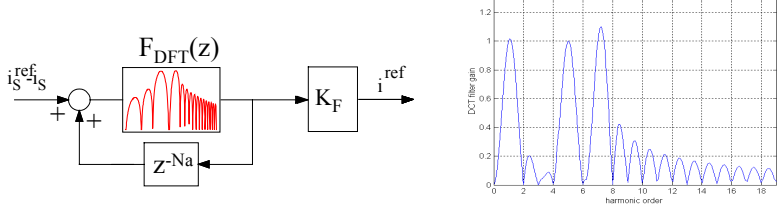

Fig. 6 - Repetitive-based control scheme and DCT frequency response for $\mathrm{N}_{\mathrm{h}}=[1,5,7]$.

\section{Compensation Strategies: Advantages and Disadvantages}

Among several possible compensation methods based on the described decompositions and digital 
techniques, four strategies are discussed to demonstrate that the same shunt active filter leads to different results using one or other method and all of them can present positive and negative aspects depending on the utility voltage conditions and the specific power quality problem to be eliminated.

1) SSC method - sinusoidal source current synthesis: As proposed in [6], when using this strategy, the currents drawn from the power system assume sinusoidal and balanced waveforms, independently of the PCC voltage waveforms. To provide balanced currents, storage elements are required.

By using the selective identification method, the reference signals for an active filter are provided in a different way that was originally proposed. Here the disturbing currents $\left(\boldsymbol{i}_{\boldsymbol{d}}\right)$ are evaluated applying sequentially the three decompositions (6), according to Fig. 2.

$$
i_{d}=i-i_{1 p}^{+}
$$

From the point of view of current deterioration, this technique is very attractive, but concerning power quality purposes, the utilization of this method may be controversial, since imposing sinusoidal and symmetrical currents with generic voltages may give raise to oscillatory power transfer.

2) RLS method - resistive load synthesis: The second strategy uses similar concepts of the Orthogonal Theory presented by Fryze and generalized in [11-13], in order to calculate the active filter control reference signals. This technique imposes balanced currents, which are proportional to the measured voltages at the PCC, resulting a resistive load, even if the voltages present distortion and small imbalances. Such strategy is named unit-power factor control in [3].

As proposed in [4], this strategy is based on applying directly the third decomposition on the measured voltages and currents (see Fig. 3). However, the authors point out some difficulty of finding a simple and fast treatment to provide the instantaneous active current, while maintaining the total power balance. Thus a slow varying current proportionality factor (average conductance) was used, originated from the average active power balance at the compensator DC link.

3) VRLS method - variable resistive load synthesis: Based on the previous strategy, this techniques uses only the third decomposition (Instantaneous Lagrange Multiplier) to emulate an instantaneous proportionality factor (conductance) based on the PCC voltages [7]. Thus, proportional currents (active) are calculated using the ratio between the instantaneous three-phase power and the instantaneous voltage norm, as described in $[11,13]$. Considering that this ratio may vary instantaneously, the proportional current $\boldsymbol{i}_{\boldsymbol{p}}$ is variable; which means that the control synthesizes a variable resistive load.

$$
\boldsymbol{i}_{\boldsymbol{p}}=\frac{v_{a} \cdot i_{a}+v_{b} \cdot i_{b}+v_{c} \cdot i_{c}}{v_{a}+v_{b}+v_{c}} \cdot \boldsymbol{v} .
$$

Since this strategy is based on an instantaneous mathematical function, its dynamic response is very fast. In addition, it can be applied to systems with any voltage and current deterioration. An active filter using this strategy does not need storage elements to maximize the power factor and reduce the system losses, however, it is not able to provide the currents balance in the same way as the previous strategy [7].

4) SHC Method - selective harmonic compensation: As recently proposed [8,9], selective harmonic compensation reduces active filter's rating, since the compensation capability is only used to eliminate the desired harmonic components and could be used to ensure that individual or total harmonic current distortion of a specific load does not exceed pre-defined recommended limits.

The selective harmonic compensation method explored in this paper is detailed in [9]. It uses the measurement of line currents and a repetitive-based control technique to achieve a very precise reference tracking in the presence of periodic distortion (see Fig. 6). In order to achieve compensation of only the selected frequencies and to provide a leading-phase needed for improving the stability margin, a modified scheme using DFT (Discrete Fourier Transform) based filters, is used. A PLL system is applied to provide frequency deviation immunity for such digital band-pass filters. Figure 7 illustrates the active filter scheme using such strategy.

\section{Simulation Results}

The power system used in the simulations is shown in Fig. 8. The SSC and RLS strategies were compared, considering two different conditions at a general PCC.

The shunt active filter was connected in order to compensate the non-linear behavior of the load side, represented by a three-phase inductive linear load, operating in parallel with three-phase and single-phase

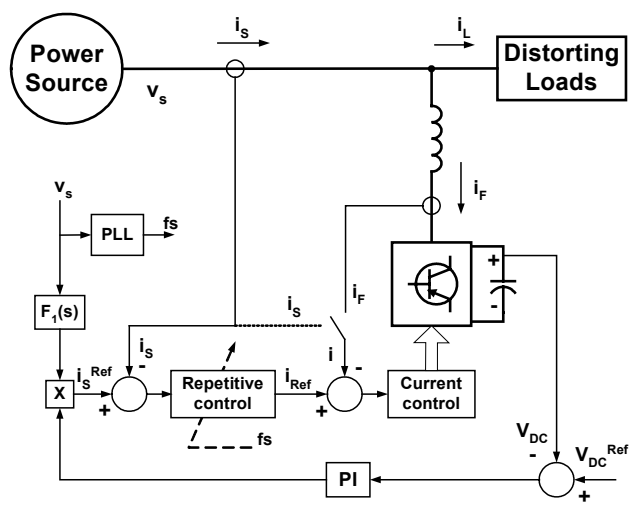

Fig. 7 - Active filter using selective harmonic compensation based on repetitive control. 


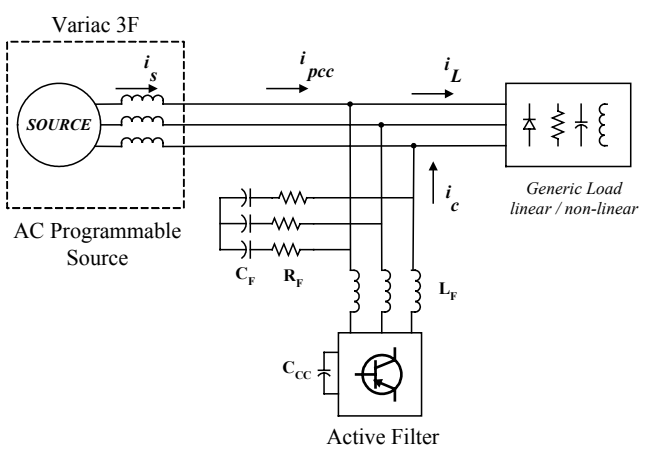

Fig. 8 - Evaluated power system.

diode rectifiers. Particularly in the first two simulations, an additional three-phase rectifier was connected to the power grid with a delay of $100 \mathrm{~ms}$ for comparing the dynamic response of the active filters.

\section{A. Test Case 1}

In the first case, the utility voltages present $3 \%$ of $7^{\text {th }}$ harmonics and $4 \%$ voltage reduction of phases $b$ and $c$. Fig. 9 represents the PCC compensation based on the SSC method, using the instantaneous decompositions to eliminate the global disturbing currents $\left(\boldsymbol{i}_{\boldsymbol{d}}\right)$.

After compensation, the PCC currents are sinusoidal and balanced and the power factor results near unity. When the second rectifier is connected, it is also possible to notice the fast dynamic response of this strategy, which in this case was implemented by means of the DCT filter (first decomposition), the instantaneous Fortescue's transformation (second decomposition) and the Lagrange Multiplier.

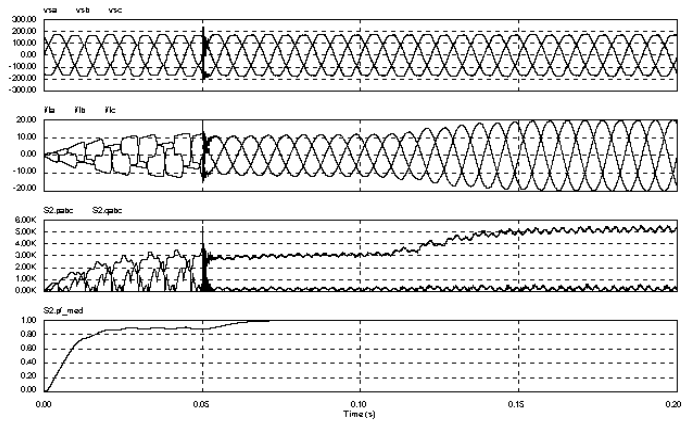

Fig. 9 - CASE 01: Instantaneous PCC voltages, currents, active and non-active powers and power factor-SSC Method

Such technique is very efficient for harmonic, unbalanced and non-active currents compensation, even when the PCC voltages are distorted and/or unsymmetrical. Nevertheless, since the voltages and currents have different frequency compositions, the active power may be oscillatory and there is no damping effects related to any possible system resonance.

For the same case, Fig. 10 presents the PCC compensation using the RLS method (directly applying the Lagrange Multiplier). Since the source voltages are rather sinusoidal and balanced, the compensated currents are almost balanced and proportional to the voltages and the power factor is also increased to unity.

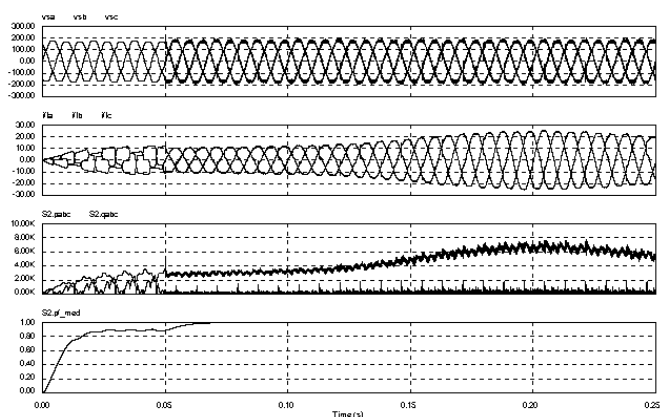

Fig. 10 - CASE 01: Instantaneous PCC voltages, currents, active and non-active powers and power factor-RLS Method

\section{B. Test Case 2}

In the second case, a three-phase linear inductive load with a power factor correction capacitor in parallel, were connected between the active filter PCC and the power source sides. This case represents a very common situation in distribution systems, especially in industrial utilities.

The capacitor value $(145 u F)$ was intentionally chosen in order to create a resonance frequency along with the line impedances $(1 \mathrm{mH})$ at the $7^{\text {th }}$ harmonic, which is present in the source voltages and also in the PCC load currents. This situation was simulated in order to demonstrate the different effects of each type of filter control strategy in such resonant condition.

In order to show that the source voltages were distorted by the passive resonance even without the PCC's loads, these were connected just $20 \mathrm{~ms}$ after the simulation beginning. Notice in Fig. 11 (SSC method) that when the non-linear loads were connected, a small increase in the resonance effect is observed due to the rectifiers behavior.

Fig. 11 shows the compensation results using the Modified SSC method for this case. After compensation, the current waveforms are sinusoidal and balanced and the power factor is improved near to unity. However, this strategy does not provide any damping to the voltage distortion.

This is because the pure sinusoidal currents, imposed in the presence of disturbed voltages, force the system to sense the load exclusively at the fundamental frequency. At other frequencies the load behaves as infinite impedance, since no current will flow at these frequencies. In the very common case of power factor capacitors connection near the active filter, at least one resonant frequency will be defined. Thus the lack of damping of this frequency may lead to a dangerous condition concerning PCC voltage resonance.

From Fig. 12 it is possible to observe that if the active filter using the RLS method is inserted (at $50 \mathrm{~ms}$ ), the resistive load emulation is capable to improve the 


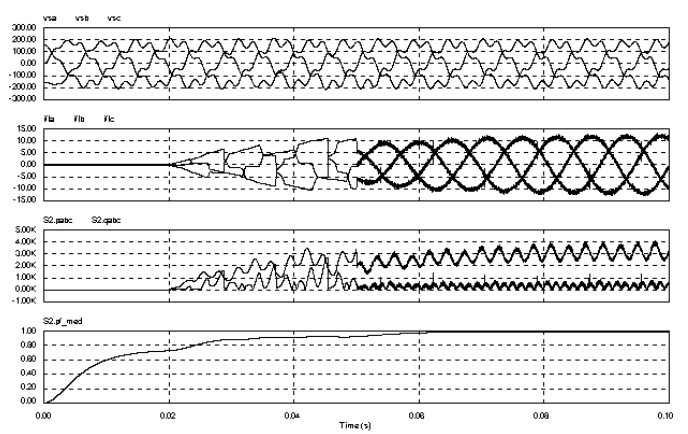

Fig. 11 - CASE 02: Instantaneous PCC voltages, currents, active and non-active powers and power factor-SSC Method

current waveform and also the power factor. The increased damping effect provides a significant improvement in the supply voltages.

However, if the standard limits to the current distortion (THD) were used as charging factors by the utilities, this technique could lead to penalty charges to the consumers if the voltage deterioration were significant.

The previous results show that it is very important to analyze those differences and to decide when to use one or another technique to solve a general or a specific power quality problem, since it is clearly impossible to achieve all advantages of each strategy in a single shunt active filter. Moreover, the flexibility of selective compensation may be very attractive for power conditioners development, since it enables easy changes from one to another compensation strategy.

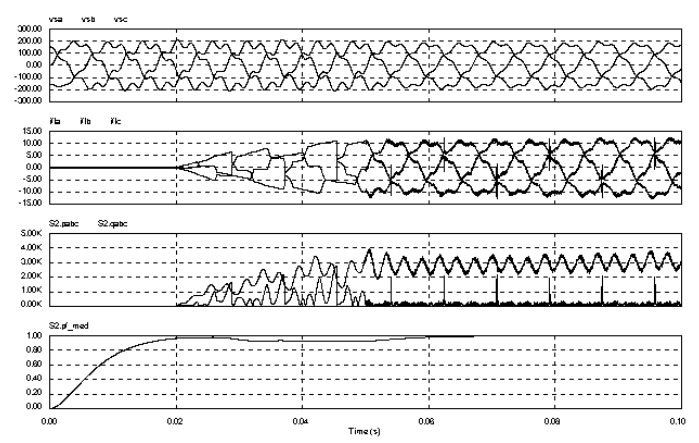

Fig. 12 - CASE 02: Instantaneous PCC voltages, currents, active and non-active powers and power factor-RLS Method

\section{Experimental Results}

In order to validate the previous discussions, next experimental results were obtained using a $5.5 \mathrm{kVA}$ converter, a fixed-point DSP-based system (ADMC401) and a Hall effect sensor board (LV25P and LA55P/SP1). Table I shows the parameters used in the practical implementation.

The first verification was done on the harmonic compensation of an uncontrolled rectifier. Using the SSC Method and a PI current control, Fig. 13 shows the phase voltage, source $i_{S}$ and load $i_{L}$ currents, respectively. The spectra of the line currents are also reported. Note that
Table I - Power system and control parameters.

\begin{tabular}{|l|l|}
\hline DC Link Voltage & $450 \mathrm{~V}$ \\
\hline Filter Inductor $\mathrm{L}_{\mathrm{F}}$ & $4 \mathrm{mH}$ \\
\hline Switching Frequency & $12 \mathrm{kHz}$ \\
\hline Line voltage & $220 \mathrm{~V}_{\text {rms }}$ \\
\hline Mains frequency & $60 \mathrm{~Hz}$ \\
\hline Nominal Load Power & $5 \mathrm{kVA}$ \\
\hline Selected frequencies $(h)$ & $1^{\text {st }}, 3^{\text {rd }}, 5^{\text {th }}, 7^{\text {th }}, 9^{\text {th }}, 11^{\text {th }}, 13^{\text {th }}, 17^{\text {th }} \ldots 29^{\text {th }}$ \\
\hline Current control definition & $1 \mathrm{kHz}$ bandwidth, $70^{\text {o }}$ phase margin \\
\hline
\end{tabular}

there is a small attenuation of the load harmonics. This is mainly due to the delay of the digital current control, which deteriorates the performance of the SSC strategy. This shows that, it is not always possible to achieve the theoretical expectations in practical implementations. In this condition, the load current THD is $24.9 \%$, while the source current THD using the SSC with PI control is reduced to $12.3 \%$, which is still significantly high. In such case, the SSC was implemented using the novel Positive Sequence Detector and the Lagrange Multiplier.

As a comparison, we have tested the proposed $S H C$ control in the same conditions and the results are reported in Fig. 14. The compensation of all odd harmonics up to the $29^{\text {th }}$ harmonic was imposed and it is possible to note an improvement in the line current waveforms. Looking at the spectrum of line currents in this condition, it is possible to note that all selected frequencies have been well compensated, as theoretically foreseen. So, the THD is reduced to less than $2 \%$, which attends most of international recommendations and standards.

The resulting current waveform in this former case will be much more sinusoidal than using directly the SSC method with PI current controller, but in this case using all the advantages of the selective harmonic compensation method.

\section{Conclusion}

This paper has discussed diverse compensation strategies and digital processing techniques for practical implementations. Moreover, an alternative methodology to generate the active filter references is presented.

Such method allows identifying selectively the disturbing components of voltage and current signals and the most attractive feature of such approach is the capability to explore the compensation of specific disturbing effects, thus concentrating the application to the local necessity or economical viability.

Advantages and disadvantages of four control strategies were also discussed focusing power quality issues.

In addition, in order to validate the theoretical expectations, simulation and experimental results have demonstrated how important is the correct choice of the compensation strategy and how the applied digital algorithms can affect the active filter's dynamical and steady state performance. 


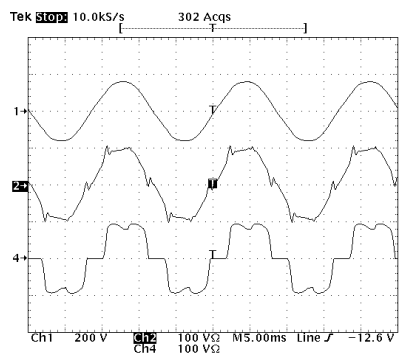

(a)

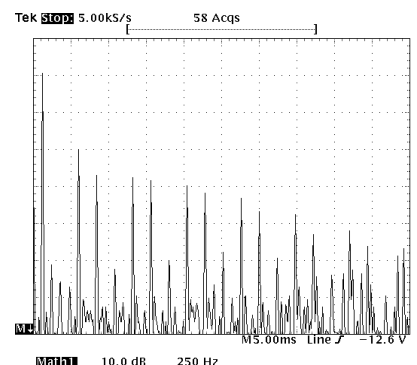

(b) (a) Line voltage $\mathrm{v}_{S}(200 \mathrm{~V} / \mathrm{div})$, line current $i_{S}(10 \mathrm{~A} / \mathrm{div})$ and load current $i_{L}(10 \mathrm{~A} / \mathrm{div})$.

(b) Spectrum of the compensated source currents (10dB/div $-250 \mathrm{~Hz} / \mathrm{div})$.

Fig. 13 - Active filter using SSC and PI current control.

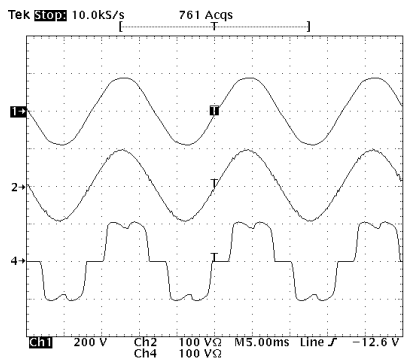

(a)

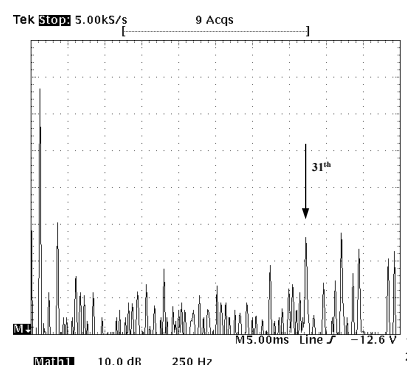

(b) (a) Line voltage $\mathrm{v}_{S}(200 \mathrm{~V} / \mathrm{div})$, line current $i_{S}(10 \mathrm{~A} / \mathrm{div})$ and load current $i_{L}(10 \mathrm{~A} / \mathrm{div})$.

(b) Spectrum of the compensated source currents (10dB/div $-250 \mathrm{~Hz} / \mathrm{div})$.

Fig. 14 - Active filter using $S H C$ and repetitive control.

\section{Acknowledgement}

Support for this research comes from FAPESP Foundation (99/11882-5) and Analog Devices.

\section{References}

[1] F.Z. Peng "Harmonic Sources and Filtering Approaches" IEEE Industry Applications Magazine, vol. 7, no. 4, July/Aug. 2001, pp. 18-25.

[2] H. Akagi, S. Ogasawara, H. Kim, "The theory of instantaneous power in three-phase four-wire systems: A Comprehensive Approach", IEEE Industry Application Society Annual Conference, 1999, pp.431-439.

[3] A. Cavallini and G. C. Montanari, "Compensation Strategies for Shunt Active-Filter Control", IEEE Trans. on Power Electronics, vol. 9, no. 6, 1994, pp. 587-593.

[4] T. E. N. Zuniga and J. A. Pomilio: "Shunt Active Power Filter Synthesizing Resistive Load". IEEE Trans. on Power Electronics, vol. 17, no. 2, March 2002.

[5] P. Mattavelli and P. Tenti, "High Performance Active Filters using Selective Harmonic Control", in Proc. 2000 IEEE Power Engineering Society Summer Meeting, pp. 977-982.

[6] M. Aredes, J. Häfner, K. Heumann, "Three-Phase FourWire Shunt Active Filter Control Strategies", IEEE Trans. on Power Electronics, Vol. 12, No. 2, 1997, pp. 311-318.

[7] F. P. Marafão, S. M. Deckmann, J. A. Pomilio and R. Q. Machado, "Selective Disturbing Compensation and
Comparisons of Active Filtering Strategies", IEEE International Conference on Harmonics and Quality of Power, Brazil, October, 2002, ISBN 0-7803-7672-2.

[8] G. Casaravilla, A. Salvia, C. Briozzo, E. Watanabe, "Selective Active Filter Comparison: Shunt or Shunt Hybrid with Remote Harmonic Distortion Control", Proc. of the $7^{\text {th }}$ Brazilian Power Electronics Conf (COBEP03), Fortaleza, Brazil, September 2003, pp. 877-884.

[9] F.P. Marafão, P. Mattavelli, S. Buso, S.M. Deckmann, "Repetitive-Based Control for Selective Active Filters using Discrete Cosine Transform", 7th Brazilian Power Electronics Conf (COBEP03), Fortaleza, Brazil, 2003.

[10] Buso, S., Malesani, L., Mattavelli, P.: "Comparison of Current Control Techniques for Active filter Applications", IEEE Trans. On Industrial Electronics, vol. $45, n^{\circ} 5$, October 1998, pp. 722-729.

[11] J. L. Willems, "A New Interpretation of the Akagi-Nabae Power Components for Non-sinusoidal Three-Phase Situations", IEEE Trans. on Instrumentation and Measurement, vol. 41, no. 4, pp. 523-527, 1992.

[12] L. Rosseto and P. Tenti, "Evaluation of instantaneous power terms in multi-phase systems: techniques and applications to power-conditioning equipment", European Transaction Electrical Power Engineering, vol. 4, no. 6, pp. 469-475, 1994.

[13] F.P. Marafão and S.M. Deckmann, "Basic Decompositions for Instantaneous Power Components Calculation," in IEEE Industry Applications Conference-Induscon, 2000, Brazil, pp. 750-755.

[14] A.E. Emanuel, "Apparent power: components and physical interpretation", IEEE International Conference on Harmonics and Quality of Power (ICHQP), Greece, 1998.

[15] M. Depenbrock, V. Staudt and H. Wrede, "A Theoretical Investigation of Original and Modified Instantaneous Power Theory Applied to Four-Wire Systems", IEEE Transaction on Industry Application, Vol. 39, No. 4, July/August 2003, pp. 1160-1167.

[16] L.M. Tolbert, Y. Xu, F.Z. Peng, J. Chen, J.N. Chiasson, "Definitions for Non-Periodic Current Compensation", European Power Electronics Conference (EPE), 2003, ISBN: 90-75815-07-7.

[17] F.P. Marafão, "Contributions to the Instantaneous Power Theory and its Applications in Power Quality", MSc. Dissertation, University of Campinas, Brazil, March 2000, (in Portuguese).

[18] S.M. Deckmann, F.P. Marafão, "Time based decompositions of Voltage, Current and Power Functions", IEEE International Conference on Harmonics and Quality of Power, 2000, pp. 289-294.

[19] F.P. Marafão, S.M. Deckmann, J.A.G. Marafão, "Power Factor Analysis under Non-Sinusoidal and Unbalanced Systems", IEEE International Conference on Harmonics and Quality of Power (ICHQP), Brazil, 2002.

[20] F.P. Marafão, S.M. Deckmann, A. Lopes, "Robust Delta Operator-Based Discrete Systems for Fixed-Point DSP Implementations", IEEE Applied Power Electronics Conference and Exposition (APEC), USA, February 2004.

[21] G. Andria, L. Salvatore, "Inverter drive signal processing via DFT and EKF", IEE Proceedings, Vol. 137-B, No. 2, March 1990, pp. 111-119.

[22] F.P. Marafão, S.M. Deckmann, E.K. Luna, "A Novel Frequency and Positive Sequence Detector for Utility Applications and Power Quality Analysis”, International Conference on Renewable Energies and Power Quality (ICREPQ), Barcelona, Spain, March 2004.

[23] E. Kreyszig, Advanced Engineering Mathematics, John Wiley \& Sons, Inc., eighth edition, 1999.

[24] A.V. Oppenheim, R. W. Schafer and J. R. Buck, DiscreteTime Signal Processing, Prentice Hall Inc, 1999. 